\title{
Generation bidding game with potentially false attestation of flexible demand
}

\author{
Yuquan Shan ${ }^{1}$, Jayaram Raghuram², George Kesidis ${ }^{1,2^{*}}$, David J Miller ${ }^{2}$, Anna Scaglione ${ }^{3}$, Jeff Rowe ${ }^{4}$ \\ and Karl Levitt ${ }^{4}$
}

\begin{abstract}
With the onset of large numbers of energy-flexible appliances, in particular plug-in electric and hybrid-electric vehicles, a significant portion of electricity demand will be somewhat flexible and accordingly may be responsive to changes in electricity prices. In the future, this increased degree of demand flexibility (and the onset of only short-term predictable intermittent renewable supply) will considerably exceed present level of uncertainty in day-ahead prediction of assumed inelastic demand. For such a responsive demand idealized, we consider a deregulated wholesale day-ahead electricity marketplace wherein bids by generators (or energy traders) are determined through a Nash equilibrium via a common clearing price (i.e., no location marginality). This model assumes the independent system operator (ISO) helps the generators to understand how to change their bids to improve their net revenue based on a model of demand-response. The model of demand-response (equivalently, demand-side bidding day ahead) is based on information from load-serving entities regarding their price-flexible demand. We numerically explore how collusion between generators and loads can manipulate this market. The objective is to learn how to deter such collusion, e.g., how to set penalties for significant differences between stated and actual demand, resulting in higher energy prices that benefit certain generators.
\end{abstract}

Keywords: Smart grids; Demand response; False demand attestation

\section{Introduction}

Game-theoretic approaches to the study of electricity markets, particularly under deregulation, have been explored for decades [1-3]. Recently, problems associated with variations of the optimum power-flow (OPF) problem $[4,5]$ have been considered by several authors for price-elastic demand, e.g., [6,7]. Indeed, demand elasticity for electricity is motivated by the onset of potentially enormous load from flexible appliances, particularly plugin electric and hybrid-electric vehicles, see, e.g., $[8,9]$ and the references therein, where an electric vehicle represents electricity demand comparable to the rest of the household combined.

In typical models of 'two-settlement' (wholesale dayahead and real-time) electricity markets commonly used

\footnotetext{
*Correspondence: gik2@psu.edu

${ }^{1}$ Department of Computer Science and Engineering, The Pennsylvania State University, University Park, State College, PA 16802, USA

${ }^{2}$ Department of Electrical Engineering, The Pennsylvania State University, University Park, State College, PA 16802, USA

Full list of author information is available at the end of the article
}

in practice, day-ahead settlements largely account for the real-time supply. In real time, relatively minor adjustments are expected to be made to meet actual current demand. However, significant power supply may need to be quickly secured if there are failures in supply or if realtime (actual) demand is unexpectedly different than that predicted day-ahead, e.g., due to weather conditions. On the other hand, excess supply may have been secured if elastic demand is deferred in real time, or (often intermittent and confidently predictable only in the short-term) renewable supply is employed. So, in the future, actual real-time demand may be additionally difficult to predict day-ahead.

To account for significant flexible demand in the dayahead market $[10,11]$, we assume that the load-serving entities (or demand aggregators or just consumers) will inform the independent system operator (ISO) regarding their flexible demand - a kind of demand-side bidding. The ISO is assumed to provide sufficient information so

\section{Springer}

(c) 2015 Shan et al.: licensee Springer. This is an Open Access article distributed under the terms of the Creative Commons Attribution License (http://creativecommons.org/licenses/by/4.0), which permits unrestricted use, distribution, and reproduction in any medium, provided the original work is properly credited. 
that the generators (suppliers) can modify their prices to improve upon their net utility, i.e., the ISO determines generation allocation sensitivities to bid prices of the OPF with demand response. In our setting, the ISO solves an OPF which minimizes the common clearing price of supply to the loads. The game is a 'discriminatory' sealedbid auction in that the generators earn at the price they bid but in quantity determined by the ISO [1,2]. So to simplify matters herein, we do not consider strategic bidding by the generators wherein they may infer demand and/or the bidding strategies of their competitors via a probabilistic model, nor multipart bidding to account for start-up/ramp-up costs, secure contracts involving minimum and maximum supply per generator, and the like ${ }^{\mathrm{a}}$, nor peak-power consumption penalties [12,13]. Also, we assume each generator has a continuously differentiable and convex cost of supply [3], quadratic in particular [14]. Our model is related to noncooperative Cournot games of electricity markets reversing the direction of day-ahead markets to understand how demand responds to market clearing prices in the long term [15] - here we attempt to understand demand-response on the wholesale (generation-level) market.

How an energy trader (or load-serving entity colluding with one or more generators) can manipulate day-ahead electricity prices by placing uneconomic demand-side bids is studied in $[16,17]$, not considering flexible demand. In this paper, we are interested in how the wholesale dayahead market can be manipulated by colluding generators and load-serving entities, through misrepresentation of flexible demand by the latter.

This paper is organized as follows. We first specify our model of wholesale electricity market under demand response as a noncooperative game - an argument for the existence of its symmetric Nash equilibrium is given in the Appendix. We then use this model to numerically consider the effects of demand misrepresentation for the example of the benchmark IEEE 14-bus power system. The paper concludes with a summary and discussion of how such computations can inform penalties of significant discrepancies between attested and actual demands, causing the benefit of certain generators or energy traders.

\section{Problem setup}

Consider an ISO (single commodity retailer) with generators (suppliers) and loads (consumer demands). As in [6], supposing that each generator $g \in G$ sets its own price $p_{g} \$$ per kWh (or other energy commodity units). We model aggregate load to be linear in response to clearing price, $P^{\mathrm{b}}$

$$
D(P)=\left(D_{\max }-D_{\min }\right)\left(1-\frac{P}{P_{\max }}\right)+D_{\min }
$$

where $D_{\min }$ represents inflexible demand. Note that this is the simplest model of aggregate-demand flexibility and is commonly used in the literature. Although other more sophisticated model may be developed to fit cases in reality, such as plug-in electric and hybrid-electric vehicles, capturing flexibility of real-world aggregate loads is not a contribution of this paper.

Suppose that generators suffer quadratic cost of supply $[14]^{\mathrm{c}}$, so that the net utility/revenue ${ }^{\mathrm{d}}$ of the $g^{\text {th }}$ generator is

$$
u_{g}(\underline{p})=p_{g} S_{g}(\underline{p})-c_{g}\left(S_{g}(\underline{p})\right)=p_{g} S_{g}(\underline{p})-a_{g} S_{g}^{2}(\underline{p}),
$$

i.e., different generators having different $a_{g}$ parameters.

For noncooperative generator duopoly (two-players), discriminatory, single-part game with no distribution losses or constraints, and assuming that $p_{1} \neq p_{2}$ near the interior Nash equilibria, we can find surprisingly complex plurality of Nash equilibria in closed form [18].

We assume that supply allocations are the result of the optimization of a supply network by a linear program. In electricity markets, the retailer (ISO) is sometimes also the distribution system.

Consider power flow in a power system with the generator set $G$, load set $L$, bus set $B$ and branch set $R$.

- Let $G_{b} \subset G$ be the set of generators on bus $b$, each having generated power $S_{g}$, price per unit supply $p_{g}$, and minimum and maximum supply $S_{g}^{(\min )}$ and $S_{g}^{(\max )}$, respectively.

- Let $L_{b} \subset B$ be the set of loads on bus $b$, each having a demand $D_{l}$ that depends on the clearing price $P$.

- For each bus $b \in B, \theta_{b}$ is its voltage angle.

- Finally, let $r_{i, j}$ be the branch connecting bus $b_{i}$ and bus $b_{j}$, with $x_{i, j}$, the reactance of the branch, $P_{i, j}$, the power 'flowing' from $b_{j}$ to $b_{i}$ (if we neglect power loss on the transmission line, we get $\left.P_{i, j}=-P_{j, i}\right)$, and $c_{i, j}$, the maximum tolerable power on the branch.

\section{Optimal power flow problem formulation}

Assuming fixed generation prices $p$, and associated clearing price $P$, the total load (consumer demand) is given by $D(P)$. The individual loads are assumed to be some fixed proportion of the total demand, i.e., $D_{l}(P)=\alpha_{l} D(P), \forall l \in$ $L$, where $\alpha_{l}>0$ and $\sum_{l \in L} \alpha_{l}=1$. The ISO solves a constrained optimization problem (linear programming in this case) in order to find $\underline{S}=\left[S_{1}, \ldots, S_{|G|}\right]$, the optimum power generating assignment for the generators, and $\underline{\theta}=$ $\left[\theta_{1}, \ldots, \theta_{|B|}\right]$, the voltage angles on the buses, which mini- 
mize the cost charged by the generators. The constrained optimization problem is thus given by:

$$
\min _{\underline{S}, \underline{\theta}} \sum_{g \in G} p_{g} S_{g}
$$

such that:

$$
\begin{aligned}
& \left.P_{i, j}=\frac{1}{x_{i, j}}\left(\theta_{j}-\theta_{i}\right) \quad \text { (DC approximation }[4]\right) \\
& \sum_{g \in G_{b}} S_{g}-\sum_{l \in L_{b}} D_{l}=\sum_{k \in B} P_{k, b}, \forall b \in B \quad \text { (power balance) } \\
& S_{g}^{(\min )} \leq S_{g} \leq S_{g}^{(\max )}, \forall g \in G \quad \text { (generator limits) } \\
& -c_{i, j} \leq P_{i, j} \leq c_{i, j}, \forall i, j \in B \quad \text { (branch limits) }
\end{aligned}
$$

Here, neglecting the power loss in generation and transmission, we have

$$
\sum_{g \in G} S_{g}=\sum_{l \in L} D_{l}
$$

Note that when all the generators have the same prices, there exist infinitely many optimal solutions in the above mathematical program. In this case, without violating other constraints, we equally divide allocation to each generator. We will pay particular attention to this situation in the following.

In the above formulation, we used fixed upper and lower bounds on the supply allocations $S_{g}$. In addition, the quadratic penalty term in the utility function (2) serves to model cost of supply allocation [3,14]. For a positive $a_{g}$ and fixed generator prices $p$, suppose generator $g$ wants to ensure that its utility function is never smaller than some positive value $u_{g}^{\text {(min) }}$, then this imposes lower and upper bounds on its supply allocation, given by

$$
\begin{aligned}
& \frac{1}{2 a_{g}}\left(p_{g}-\sqrt{p_{g}^{2}-4 a_{g} u_{g}^{(\min )}}\right) \leq S_{g} \\
& \leq \frac{1}{2 a_{g}}\left(p_{g}+\sqrt{p_{g}^{2}-4 a_{g} u_{g}^{(\min )}}\right),
\end{aligned}
$$

provided the generator price satisfies the condition $p_{g} \geq$ $2 \sqrt{a_{g} u_{g}^{(\min )}}$. If $u_{g}^{\min }=0$, then we observe that as $a_{g}$ is made larger, i.e., as the cost of supply allocation increases, the maximum supply allocation (or capacity) decreases, and vice versa.

In practice for power-transmission circuits, thermal losses may determine edge (transmission line) capacities and costs, the latter typically in a power-flow dependent fashion, e.g., ' $I^{2} R$ ' losses (Section 3.1 of [4]). In order to focus on the bidding behavior among the generators, we neglect the power loss on the transmission lines; hence, the cost in power transmission is also neglected, as in the DC approximation.

\section{Setup of generators' iterative game on a platform of demand response}

We neither assume that each generator's cost of production is known to the ISO (i.e., the $a_{g}$ terms), nor, equivalently, that the ISO chooses its allocations to the generators based on this (as in [6]). Besides, the cost function (hence the payoff function) of any generator is opaque to all its competitors, concerning this, our game can be regarded as an incomplete information game. In the following, denote as $\underline{S}(\underline{D}(P), p)$ the solution of the above optimal power flow allocation problem to determine supply allocations for fixed demands (which are based on the clearing price $P) \underline{D}(P)=\left\{D_{l}(P) \mid l \in L\right\}$ and fixed generator prices $\underline{p}=\left\{p_{g} \mid g \in G\right\}$. We propose the following iterative generator game wherein, for fixed generator prices, the clearing price and the consumer demands are adjusted iteratively until they converge to a fixed point. Then each generator $g \in G$ adjusts its price $p_{g}$, given the current price for all other generators $\underline{p}_{-g}$, such that its utility function $u_{g}\left(p_{g}, \underline{p}_{-g}\right)$ is increased. Given initial prices set by the generators $\underline{p}$, the iterative generator game proceeds as follows:

1. The ISO sets an initial mean price of supply (clearing price charged to all consumers), $P$, say just as the mean of the initial generator prices, $p_{g}, \forall g \in G$.

2. Determine the price-dependent loads $\underline{D}(P)$, where $D_{l}(P)=\alpha_{l} D(P), \forall l \in L$.

3. ISO solves the optimal power flow allocation problem $\underline{S}(\underline{D}(P)), \underline{p})$ given fixed demands $\underline{D}$ and generation/supply costs $p$.

4. ISO computes a new mean (clearing) price of supply, $P=\frac{\sum_{g \in G} S_{g} p_{g}}{\sum_{g \in G} S_{g}}$.

5. If the change in clearing price $P$ is significant (larger than some threshold), then go back to Step 2; else continue to Step 6.

6. For the current set of generator prices, consistent supply allocations, loads, and clearing price have been found. Now each generator sets a new price of supply such that there is an increase in its utility function using one of the following two approaches: (i) Best-response play action: Each generator $g$ sets a new price of supply based on (an estimate of) ${ }^{\mathrm{e}}$

$$
\arg \max _{p_{g}} p_{g} S_{g}\left(p_{g} ; \underline{p}_{-g}\right)-c_{g}\left(S_{g}\left(p_{g} ; \underline{p}_{-g}\right)\right)
$$

where $c_{g}(x)$ is the cost of supply (assumed $=a_{g} x^{2}$ above). 
(ii) Better-response play action: Each generator $g$ obtains approximate left and right partial derivatives of its utility function with respect to its price $p_{g}$, i.e.,

$$
\begin{aligned}
\Delta u_{g}^{+} & =\frac{u_{g}\left(p_{g}+\epsilon, \underline{p}_{-g}\right)-u_{g}\left(p_{g}, \underline{p}_{-g}\right)}{\epsilon} \\
\Delta u_{g}^{-} & =\frac{u_{g}\left(p_{g}, \underline{p}_{-g}\right)-u_{g}\left(p_{g}-\epsilon, \underline{p}_{-g}\right)}{\epsilon},
\end{aligned}
$$

where $\epsilon \searrow 0^{\mathrm{f}}$. If the left and right derivatives have different signs (a non-differentiable point), then there are two possibilities. If $\Delta u_{g}^{-}>0$ and $\Delta u_{g}^{+}<0$, the current price $p_{g}$ is a local maximum and there is no need to change $p_{g}$. If $\Delta u_{g}^{-}<0$ and $\Delta u_{g}^{+}>0$, the current price is a local minimum. In this case, we increase $p_{g}$ by a small value $\zeta$ if $\left|\Delta u_{g}^{+}\right|>\left|\Delta u_{g}^{-}\right|$; otherwise, we decrease $p_{g}$ by $\zeta$. In case the derivatives have the same sign (may still be a non-differentiable point), we increase $p_{g}$ by $\zeta$ if both derivatives are positive and decrease $p_{g}$ by $\zeta$ if both derivatives are negative. The step $\zeta$ should increase the price by a small value such that there is an increase in the value of the utility function. It should not make large changes to the price like the best-response play actiong.

7. Exit if there is no change in the generator prices (i.e., if an equilibrium set of prices is obtained); Else go back to Step 1.
We implicitly assume in Step 6 that the ISO helps to compute estimates of supply sensitivities to bid for each generator, $\partial S_{g} / \partial p_{g}$. This can be done numerically through difference quotients. The result is a Jacobi iteration representing a 'better response' game $[19,20]$. Alternatively, the ISO could iteratively compute the 'best response' price for each generator, but this would require knowledge of each generator's net utility.

\section{Numerical study - benchmark IEEE 14-bus power system}

We study the iterative generator game described above with the benchmark IEEE 14-bus power system that has 3 generators and 11 loads as shown in Figure 1 [21]. We consider the scenario where there is no cost/loss in delivering power, and the branch capacities are set to fixed values. Here we only focus on three-player best-response game; discussion of a better-response, two-player game for the benchmark IEEE 9-bus system, with numerical results, is given in [22].

For this 14-bus power system, each of the three generators can produce maximum power $\underline{S}_{g}^{(\max )}=150 \mathrm{MW}$, and minimum power of $0 \mathrm{MW}$ (again, we do not consider ramp-up/down issues herein). The constants in $D(P)$, our model for the total load, were chosen as the possible maximum/minimum power provided by the generators, that is, $D_{\max }=450 \mathrm{MW}, D_{\min }=0 \mathrm{MW}$. The total load $D(P)$ is assumed to be proportionally divided among the individual loads, e.g., $\alpha_{l}=\frac{1}{|L|}, \forall l \in L$. The maximum clearing

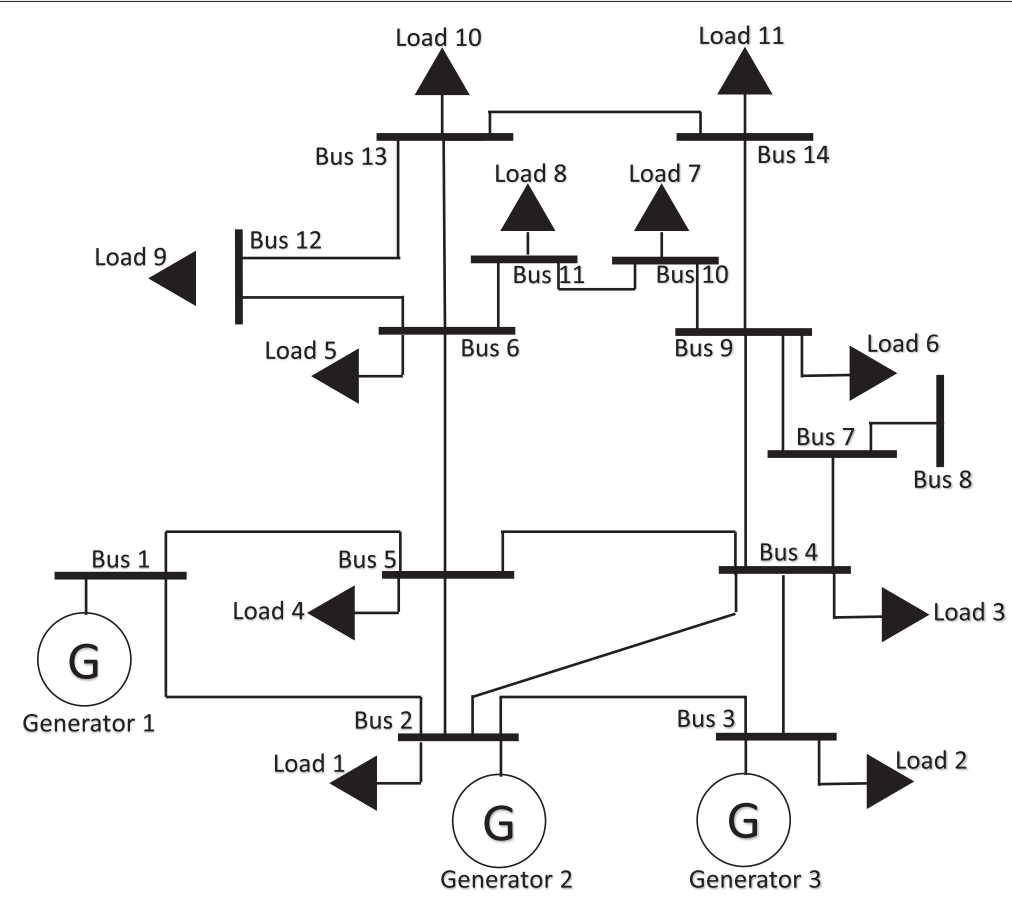

Figure 1 IEEE 14-bus power system having an asymmetric topology with 3 generators and 11 loads. 
price is set to $P_{\max }=5$; for clearing prices $P>P_{\max }$, the flexible demand is 0 . Other data for this power system can be found in [23]. The constants $\left(a_{g}\right)$ in the utility function (2) of generators are set to $0.02,0.025$, and 0.03 , respectively.

We had all three generators participating in the competition, using randomly chosen starting price-points (play-action bids). Most starting points resulted in convergence to the symmetric Nash equilibrium point $p^{*}=$ $[3.53,3.53,3.53]$ as shown in Figure 2. Additionally, we numerically found other symmetric Nash equilibrium points forming a line segment, as shown in Figure 3. In the Appendix, we prove the existence of a plurality of symmetric Nash equilibrium points and the lack of asymmetric ones, under certain conditions for our test systems. In [22], we describe a numerical study with similar results for the benchmark IEEE 9-bus power system (having a symmetric topology unlike the 14-bus system).

\section{Effects of false demand attestations}

We also numerically explored how false demand attestations may impact the revenues of generators. It is possible that demand aggregators and generators may collude, or noneconomic demand-side bids may be placed by energy brokers, to cause some generator(s) to receive more revenue while possibly reducing the revenue of others.

We first explain that by considering the convex quadratic cost model of (2) how modifying power allocations to generators may improve their revenues. In particular, we can demonstrate in some situations, decreasing power consumption could be beneficial for some generators, especially for those with relatively higher cost parameter $a_{g}$. Assume all the generators bid the same Nash equilibrium price, $p$, and equally share the aggregate demand $D(p)$. Clearly, the clearing price is also $p$ and each generator has allocation

$$
S_{g}=\frac{1}{|G|}\left[\left(D_{\max }-D_{\min }\right)\left(1-\frac{p}{P_{\max }}\right)+D_{\min }\right] .
$$

According to (2), the revenue of generator $g$ will be maximized when its allocation is

$$
S_{g}^{\prime}=\frac{p}{2 a_{g}}
$$

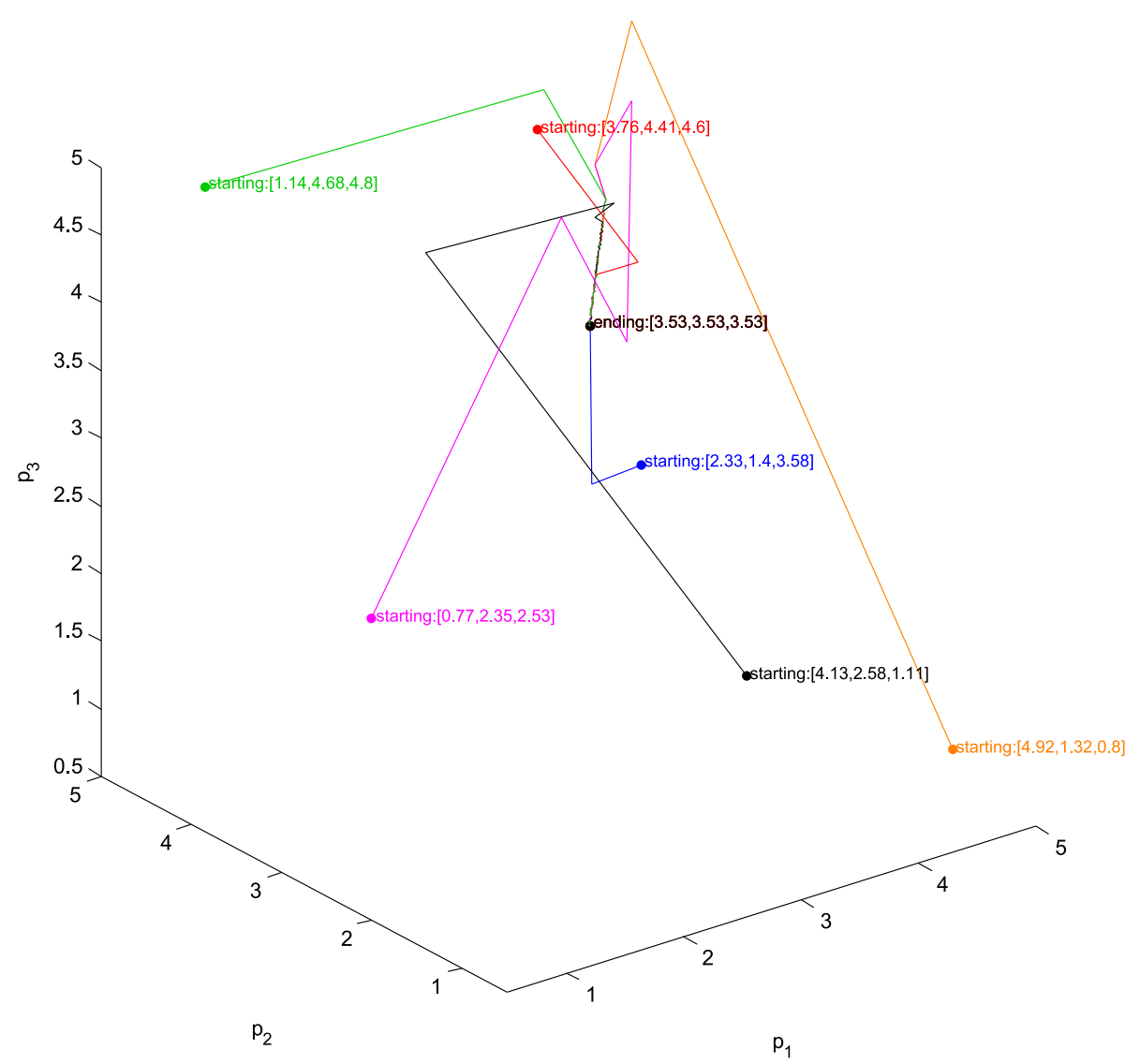

Figure 2 Sample generator-price trajectories which converge to $[3.53,3.53,3.53]$. Trajectories converge to this maximal Nash equilibrium point from most initial price points. 


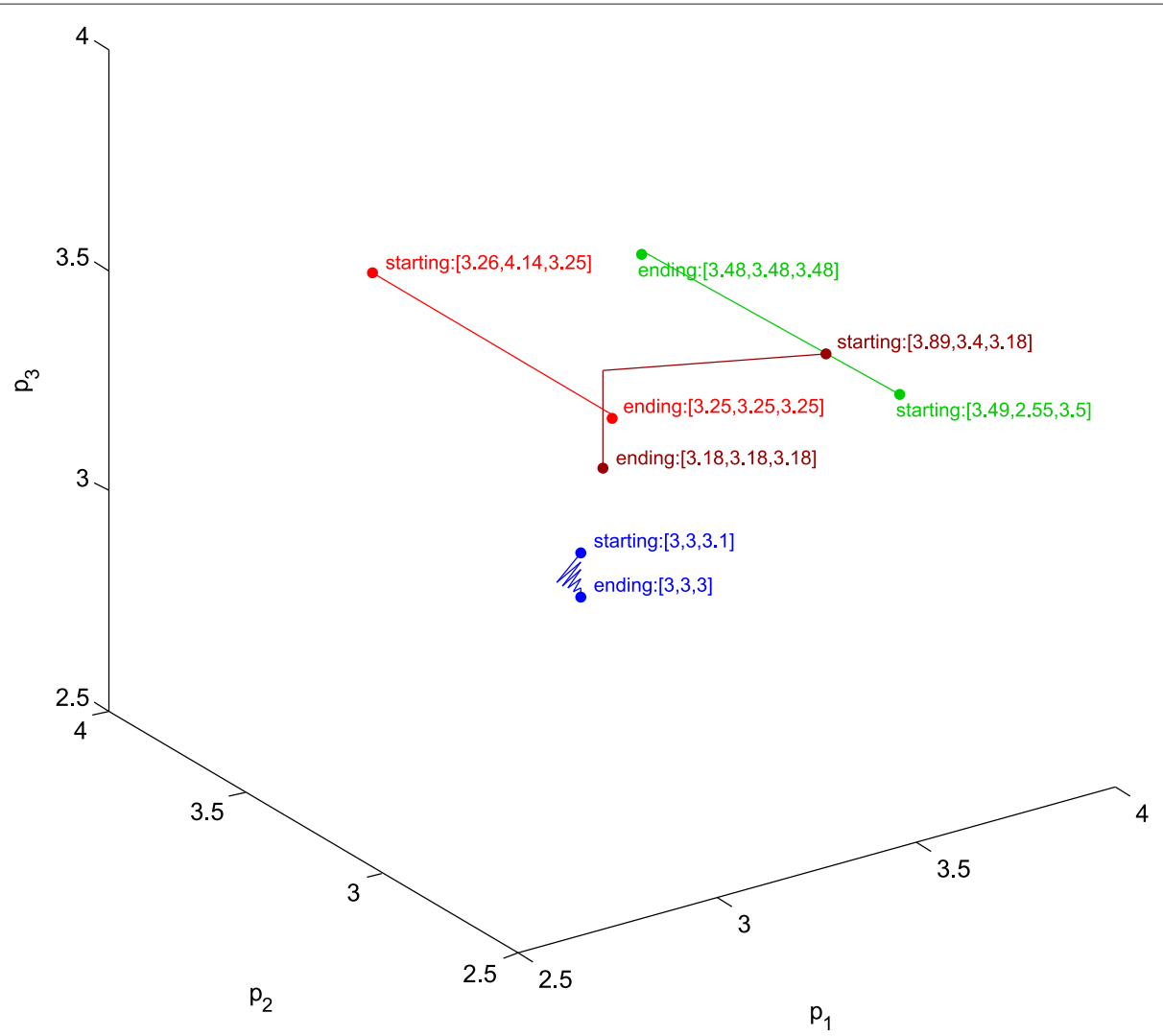

Figure 3 Sample trajectories which converge to non-maximal, symmetric Nash equilibrium points.

Thus, when the current allocation of generator $g, S_{g}$, is larger than its optimal allocation, $S_{g}^{\prime}$, less allocation brings more revenue. For the same reason, if $S_{g}<S_{g}^{\prime}$ then less allocation $S_{g}$ leads to financial loss. That is, the following inequality at symmetric Nash equilibrium $p$ will determine whether increased or reduced allocation will be beneficial to a given generator $g$ :

$$
\begin{aligned}
& S_{g}>S_{g}^{\prime} \Longleftrightarrow \frac{1}{|G|} \\
& {\left[\left(D_{\max }-D_{\min }\right)\left(1-\frac{p}{P_{\max }}\right)+D_{\min }\right]>\frac{p}{2 a_{g}}}
\end{aligned}
$$

\section{Example with numerical study}

In the benchmark IEEE 14-bus power system described above, we thereby predicted that decreasing allocation will bring generators 1,2 , and 3 more profit when the current equilibrium price is lower than $2.72,3$, and 3.21 , respectively. We verified these thresholds numerically as follows. Denote the factor by which load $j$ has modified its demand as

$$
\Gamma_{j}=\frac{D_{j, f a l s e}}{D_{j, \text { true }}} .
$$

Assume load 1 reports a false demand which is greater than its actual demand, $\Gamma_{1}>1$, while the other loads are true, i.e., $\Gamma_{i}=1, \forall i \neq 1$. False demands were used to determine prices while true demands were used when computing the generator net utilities via the power circuit.

The generation net utilities at Nash equilibrium are depicted in Figure 4 as a function of $\Gamma_{1} \geq 1$. The upperleft plot depicts the case with (maximal) Nash equilibrium prices [3.53,3.53,3.53], in which no generator benefits when $\Gamma_{1}>1$. The upper-right plot depicts the case with equilibrium prices $[3.1,3.1,3.1]$, in which only generator 1 will benefit. Lower-left plot depicts the case with equilibrium price $[2.8,2.8,2.8]$, in which only generators 2 and 3 will benefit. Finally, the lower-right depicts the case with equilibrium prices $[2.6,2.6,2.6]$, in which all the generators will benefit. The effects of false demand attestation in IEEE 9-bus power system is also studied, similar results are observed.

\section{Discussion: when to penalize}

Recall the previous discussion of complications in estimating demand day-ahead due to the presence of flexible demand, renewables, and storage devices. This additionally complicates detection of false attestations of 

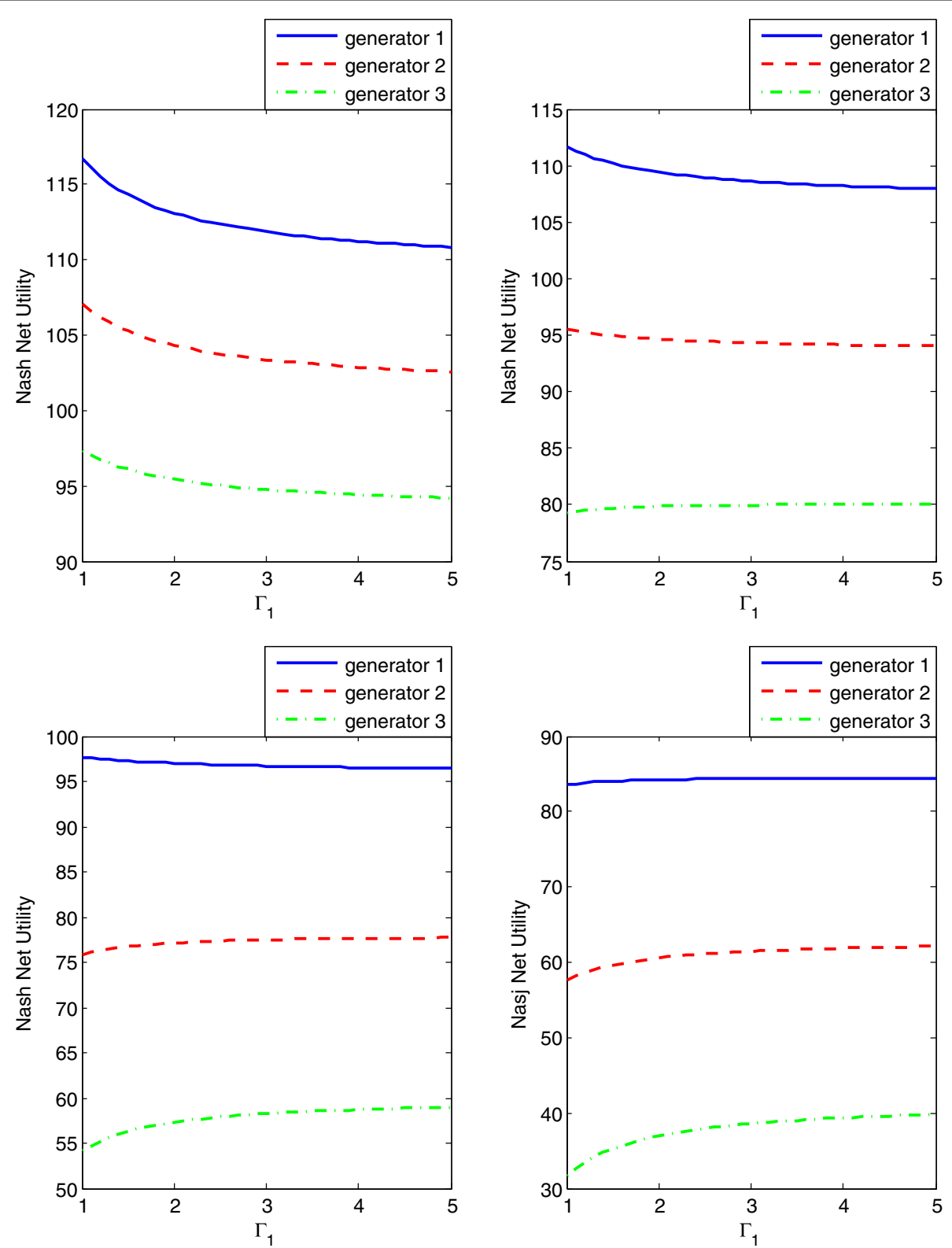

Figure 4 Nash-equilibrium net utilities vs $\Gamma_{1}$ plots under different equilibrium prices $\underline{p}$, clockwise from upper left: $\underline{p}=[3.53,3.53,3.53]$, $[3.1,3.1,3.1],[2.6,2.6,2.6],[2.8,2.8,2.8]$.

demand. So, an ISO may need to detect persistent demand-attestation 'biases' over time and let that trigger associated demand-side penalties or reservation costs. General incomplete-information game-theoretic frameworks include Bayesian games and hypergames, e.g., [24]. In both VCG and PSP auctions (e.g., [25,26]), issues of truthfulness in the disclosed bids are considered, i.e., reflecting actual demand response (by marginal valuation). More prosaic approaches simply interpolate and extrapolate from presumed honest bids (by (amount, price)) to obtain a complete estimate of other players' demand response. These frameworks are applicable to iterated (sequential) adversarial (noncooperative) games with or without leaders. Generally, estimates are greatly simplified under the assumption that player strategies are time (play-action iteration) invariant.

The magnitude of such penalties would be informed by calculations such as those described above, i.e., by determining which generators benefit and by how much. By penalizing in this way, the motivation for deliberately false demand-attestations are disincentivized. 


\section{Conclusions}

In this paper, we studied a day-ahead wholesale electricity market under demand response as a noncooperative game under certain assumptions, in particular no location marginality. We argued for the existence of symmetric Nash equilibria and numerically studied the benchmark IEEE 14-bus power system. We then considered the effects of false demand attestation and again used the 14-bus system to show how generators may (or may not) benefit by false attestation of demands. Such calculations may be used to penalize demand attestations that show persistent bias to the benefit of certain generators.

\section{Endnotes}

${ }^{a}$ For example, in [5], an affine single-part bid and associated make-whole "uplift" payments that are part of a joint integer-programming unit commitment and continuous-linear OPF (economic dispatch) problem was considered. In practice, the generation unit-commitment decisions are typically made first, e.g. simply based on mean cost of supply over the capacity range of the generator including its ramp-up costs.

${ }^{\mathrm{b}}$ Herein, just the mean price of supply - again, we assume no location marginality.

${ }^{\mathrm{c}}$ We assume quadratic cost for tractability in the duopoly studied in [18]. An alternative cost structure could be asymptotic to a maximum, e.g., $c(0) /\left(s-s_{\max }\right)$ where $c(0)$ is the cost of keeping the generator online even if zero supply is being delivered. In this paper, we do not consider ramp up/ramp-down constraints for generators.

${ }^{\mathrm{d}}$ If the net consumer utility is collectively $V(D)-P D$, then for the linear demand-response to price of (1), the utility is quadratic, concave and increasing, $V(D)=\left(P_{\max } / 2\right)\left(D_{\max }^{2}-\left(D_{\max }-D\right)^{2}\right) /\left(D_{\max }-D_{\min }\right)$ for $D_{\min } \leq D \leq D_{\max }$.

${ }^{\mathrm{e}}$ Since an explicit closed-form solution is difficult to obtain, here we simply search for objective-optimizing prices by first defining an evenly partitioned price-set from 0 to 5 .

${ }^{\mathrm{f}}$ We chose a value of $\epsilon=10^{-6}$.

${ }^{\mathrm{g}} \mathrm{We}$ chose $\zeta$ as follows. Starting with a small trial value of $\zeta=0.005 p_{g}$, if $u_{g}\left(p_{g}+\zeta, \underline{p}_{-g}\right)>u_{g}\left(p_{g}, \underline{p}_{-g}\right)$ we accept the value of $\zeta$; Else $\zeta$ is decreased by a factor of 2 iteratively until $u_{g}\left(p_{g}+\zeta, \underline{p}_{-g}\right)>u_{g}\left(p_{g}, \underline{p}_{-g}\right)$.

\section{Appendix: symmetry of Nash equilibrium points}

In this Appendix, we simply and directly derive conditions on the parameters of our power-system model (in DC approximation) for existence and symmetric Nash equilibria and nonexistence of asymmetric ones. The claims can be directly extended to non-quadratic cost functions for generation at the expense of closed-form expressions for the conditions of the claims in terms of the model parameters.

\section{Proof of existence of symmetric Nash equilibrium points}

For existence of a symmetric Nash equilibrium, we need show that $A \cap B \neq \emptyset$, where $A$ is the set of prices, $p$, in symmetric price vectors $p=[p, p, \ldots, p]$ from which decreasing any one price component (of a given generator) is not profitable (for that generator), and similarly $B$ is the set of prices in price vectors from which increasing a price component is not profitable.

\section{Decreasing a price component is not profitable, $A$}

Given a potential symmetric equilibrium price-vector $p$ with equal components $p$, for generator $i$ if $p \geq \hat{p}_{i}:=$ $D^{-1}\left(S_{i}^{\max }\right)$, i.e., the total demand $D(p) \leq S_{i}^{(\max )}$, then if generator $i$ decreases its price $p_{i}<p$, it will be assigned all of $D$ until $D>S_{i}^{(\max )}$. Since the net revenue of generator $i$ is assumed $u_{i}(p)=D(p) p-a_{i} D(p)^{2}$, i.e., quadratic in $p$, then without loss of generality we can take $D_{\min }=0$ so that generator $i$ will receive its maximum revenue $u_{i}^{\text {(max) }}$, when it bids price

$$
\tilde{p}_{i}=\frac{P_{\max }^{2}-2 a_{i} D_{\max } P_{\max }}{2\left(P_{\max }+a_{i} D_{\max }\right)} .
$$

However, $\tilde{p}_{i}$ is feasible only if $\hat{p}_{i} \leq \tilde{p}_{i} \leq p$. Therefore, for generators $i$ with $\hat{p}_{i} \leq p$, the best response $p_{i}^{*}$ by decreasing (case $A$ ) price from $p$ is

$$
u_{A, i}^{*}(p)= \begin{cases}\hat{p}_{i} S_{i}^{(\max )}-a_{i}\left(S_{i}^{(\max )}\right)^{2} & \text { if } \tilde{p}_{i}<\hat{p}_{i}\left(p_{i}^{*}=\hat{p}_{i}\right) \\ p D(p)-a_{i} D(p)^{2} & \text { if } \tilde{p}_{i}>p\left(p_{i}^{*}=p\right) \\ u_{i}^{(\max )} & \text { Otherwise }\left(p_{i}^{*}=\tilde{p}_{i}\right)\end{cases}
$$

Considering equally divided demand at $p$ among the $|G|$ generators, define $A_{i, 1}$ as follows,

$$
A_{i, 1}=\left\{p: p \frac{D(p)}{|G|}-a_{i}\left(\frac{D(p)}{|G|}\right)^{2} \geq u_{A, i}^{*}(p), \hat{p}_{i} \leq p\right\} .
$$

On the other hand, if $p<\hat{p}_{i}$, if generator $i$ decreases its price from $p$, its allocation will always be its maximum power, $S_{i}^{(\max )}$. Therefore, in this case, the optimal revenue in best response for generator $i$ is

$$
u_{A, i}^{\prime}(p)=p S_{i}^{(\max )}-a_{i}\left(S_{i}^{(\max )}\right)^{2} .
$$

Define

$$
A_{i, 2}=\left\{p: p \frac{D(p)}{|G|}-a_{i}\left(\frac{D(p)}{|G|}\right)^{2} \geq u_{A, i}^{\prime}(p), \hat{p}_{i} \geq p\right\},
$$

$A_{i}=A_{i, 1} \cup A_{i, 2}$, and $A=A_{1} \cap A_{2} \cap \ldots \cap A_{|G|}$. So, no generators will decrease their prices in the next round when they all bid $p \in A$ in the current round. 


\section{Increasing price is not profitable, $B$}

Let $S_{-i}^{(\max )}$ denote the sum of maximum capacities of all the generators except for generator $i$. Given a potential equilibrium price $\underline{p}$, if $p \leq \bar{p}_{i}:=D^{-1}\left(S_{-i}^{(\max )}\right)$, then when generator $i$ increase its price, its allocation will not immediately drop to zero. Assume generator $i$ increases its price to $p^{*}$ from $p$, and thereby its allocation drops to $S_{i}$. Again, w.l.o.g., we assume $D_{\min }=0$ and have $p^{\prime}=\left(p S_{-i}^{(\max )}+p_{i} S_{i}\right) /\left(S_{-i}^{(\max )}+S_{i}\right)$ and $S_{-i}^{(\max )}+S_{i}=$ $D_{\max }\left(1-\frac{p^{\prime}}{P_{\max }}\right)$. By simplifying we get

$$
p_{i} S_{i}=\left(S_{-i}^{(\max )}+S_{i}\right)\left(1-\frac{S_{-i}^{(\max )}+S_{i}}{D_{\max }}\right) P_{\max }-p S_{-i}^{(\max )}
$$

Combining (8) and (2), the revenue of generator $i$ is quadratic in $S_{i}$ and maximum at a point $\bar{S}_{i}$ that is independent of $p$ :

$$
\bar{S}_{i}=\frac{D_{\max }-2 S_{-i}^{(\max )}}{2\left(P_{\max }+a_{i} D_{\max }\right)} P_{\max }
$$

with maximum value $u_{i}^{(\max ) \prime}$ that's linear in $p$.

Let $S_{i}^{\circ}$ be the allocation of generator $i$, when it bids maximum price, $P_{\max }$. At potential symmetric equilibrium point $\underline{\mathrm{p}}$, if generator $i$ satisfies $p \leq \bar{p}_{i}$, its optimal revenue in best response can be expressed as

$$
u_{B, i}^{\prime}(p)= \begin{cases}\left(D(p)-S_{-i}^{(\max )}\right) p-\left(D(p)-\left(S_{-i}^{(\max )}\right)^{2}\right. & \text { if } \bar{S}_{i} \geq D(p)-S_{-i}^{(\max )} \\ u_{i}^{(\max ) \prime} & \text { if } S_{i}^{\mathrm{r}}<S_{i}<D(p)-S_{-i}^{(\max )} \\ S_{i}^{\mathrm{r}} P_{\max }-a_{i}\left(S_{i}^{\check{r}}\right)^{2} & \text { if } \bar{S}_{i} \leq S_{i}^{\mathrm{r}}\end{cases}
$$

Note that the three conditions can be expressed in terms of best-response price $p_{i}^{*}$ (recall $S_{i}^{\circ}$ is also known function of $p$ ).

Considering equal allocations at symmetric price point $\underline{p}$ as above, define

$$
B_{i, 1}=\left\{p: p \frac{D(p)}{|G|}-a_{i}\left(\frac{D(p)}{|G|}\right)^{2} \geq u_{B, i}^{\prime}(p), \hat{p}_{i} \geq p\right\} .
$$

When $p>\bar{p}_{i}:=D^{-1}\left(S_{-i}^{(\max )}\right)$, once generator $i$ increases its price, its allocation will immediately drop to zero, and so will its net revenue. So, define

$$
B_{i, 2}=\left\{p: p \frac{D(p)}{|G|}-a_{i}\left(\frac{D(p)}{|G|}\right)^{2} \geq 0, \bar{p}_{i}<p\right\} .
$$

As above, also define $B_{i}=B_{i, 1} \cup B_{i, 2}$ and $B=B_{1} \cap B_{2} \cap$ $\ldots \cap B_{|G|}$. We have therefore proven the following claim.

Claim: If generator parameters $\left\{a_{i}, S_{i}^{(\max )}\right\}_{i \in G}$ and aggregate demand parameter $D_{\max }, P_{\max }$ are such that $A \cap B \neq \emptyset$, then there exists price-symmetric Nash equilibria $p=[p, p, \ldots, p](p \in A \cap B)$ with allocation symmetry $\bar{S}_{g}=D(p) /|G|$ for all $g \in G$.

As an example of a set of parameters where such Nash equilibria exist, we refer to the benchmark IEEE 14-bus power system in DC approximation described above.

\section{Proof of nonexistence of asymmetric Nash equilibrium points}

We set $D_{\min }=0$ and the minimum power allocation of each generator to zero both w.l.o.g. Instead of simply proving nonexistence of asymmetric Nash equilibrium points for the IEEE 14-bus power system under DC approximation, we herein give the conditions for the nonexistence of asymmetric Nash equilibrium points in more general form, which is useful in analyzing the systems with more than three generators.

\section{Case of a unique generator having the lowest price}

Recall the clearing price is $P=\sum_{i} p_{i} S_{i} / \sum_{g} S_{g}$.

Claim: If there is an asymmetric equilibrium point with the unique lowest price, say $p_{i}$, then the total demand $D(P) \leq S_{i}^{(\max )}$.

Proof. For a proof by contradiction, assume a Nash equilibrium pricing point where $D(P)>S_{i}^{(\max )}$ and $p_{i}$ is the lowest price. Generator $i$ can increase its price by a small amount while maintaining lowest-price status and the condition $D(P)>S_{i}^{(\max )}$. Doing so, the allocation $S_{i}$ of generator $i$ will not be affected after the increase of price but the revenue of generator $i$ will increase. Thus, this hypothetical equilibrium pricing point is not Nash, a contradiction.

According to the claim above, in such equilibrium, only the generator $(i)$ who bids the only lowest price $\left(p_{i}\right)$ receives nonzero allocation. As a result, the price it bids is the clearing price, $P=p_{i}$. Thus, $S_{i}=D\left(p_{i}\right)$ and so we can write the revenue of generator $i$ having the lowest price as follows,

$$
\begin{aligned}
u_{i}\left(p_{i}\right)= & -\left(\frac{D_{\max }}{P_{\max }}+\frac{a_{i} D_{\max }^{2}}{P_{\max }^{2}}\right) p_{i}^{2} \\
& +\left(D_{\max }+\frac{2 a_{i} D_{\max }^{2}}{P_{\max }}\right) p_{i}-a_{i} D_{\max }^{2} .
\end{aligned}
$$

Based on (9), generator $i$ can unilaterally change its price $p_{i}$ to increase its revenue until

$$
\begin{aligned}
p_{i} & =\hat{p}_{i}=\frac{P_{\max }+2 a_{i} D_{\max }}{2 P_{\max }+2 a_{i} D_{\max }} P_{\max } \\
& =\arg \max _{p_{i}} U_{i}\left(p_{i}\right)<P_{\max }
\end{aligned}
$$

or approaches (is 'infinitely close' to) the second lowest price among generators (see the next case). 
On the other hand, given $p_{i}=\hat{p}_{i}$, the other generators currently receive no allocation and have no revenue and will consider lowering their prices $p_{j}$ for $j \neq i$ in the next round to $p_{j} \leq \hat{p}_{i}$ in the next round. Doing so, the result will be generators $i$ and $j$ will fairly share the allocation, i.e.,

$$
S_{i}=S_{j}=\frac{1}{2} D_{\max }\left(1-\frac{\hat{p}_{i}}{P_{\max }}\right)>0 .
$$

By (2), at price $\hat{p}_{i}$, generator $j$ will have positive revenue if and only if

$$
0<S_{j}<\frac{\hat{p}_{i}}{a_{j}}
$$

So, generator $j$ has no motive to reduce its price $p_{j}$ to $\hat{p}_{i}$ if

$$
\frac{1}{2} D_{\max }\left(1-\frac{\hat{p}_{i}}{P_{\max }}\right) \geq \frac{\hat{p}_{i}}{a_{j}} \Longleftrightarrow\left(\frac{a_{j}}{2}-2 a_{i}\right) \geq \frac{P_{\max }}{D_{\max }}
$$

Since condition (11) does not hold for all $i \neq j$ (because the right-hand side is always negative) for our IEEE 14bus power system under DC approximation, when $\underline{a}=$ $[.02, .025, .03])$, asymmetric Nash equilibria with unique lowest prices do not exist.

\section{Proof of the nonexistence of asymmetric Nash equilibrium point with > 1 generators at the lowest price}

Let generators $i \in M \subset G$ have the lowest price $\hat{p}$.

Claim: If there is an asymmetric equilibrium point with $|G|>|M| \geq 2$, the total demand $D(P) \leq$ $|M| \min _{i \in M}\left\{S_{i}^{(\max )}\right\}$ where the clearing price $P=p_{i}$ for all $i \in M$.

Proof. Similar to the proof for the first claim, if $D(P)>$ $|M| \min _{i \in M}\left\{S_{i}^{(\max )}\right\}$ and $S_{i}^{(\max )}=\min _{j \in M}\left\{S_{j}^{(\max )}\right\}$, then $i$ will increase its utility by increasing its price a small amount without reducing its allocation, which makes this price point not Nash.

Again, this claim means that $S_{k}=0$ and generator $k$ has zero revenue for all $k \in M$. As with (10), assuming equal allocations among all generators $i \in \mathrm{M}$, all generators $i \in M$ are 'satisfied' with nonnegative allocations if

$$
\frac{1}{|M|} D_{\max }\left(1-\frac{\hat{p}}{P_{\max }}\right) \leq \min _{i \in M} \frac{\hat{p}}{a_{i}} .
$$

To give any generator $k \notin M$ no motive to reduce their price to $\hat{p}$, we require

$$
\frac{1}{|M|+1} D_{\max }\left(1-\frac{\hat{p}}{P_{\max }}\right) \geq \frac{\hat{p}}{a_{k}} .
$$

Combining (12) and (13), an asymmetric Nash equilibrium, with more than one generator with minimal price, exists if

$$
\min _{k \notin M} a_{k} \geq \frac{|M|+1}{|M|} \max _{i \in M} a_{i} .
$$

Note that (14) is necessary but not sufficient for existence of asymmetric Nash equilibrium.

Again, for our IEEE 14-bus power system under DC approximation, if utility cost parameters $\left[a_{1}, a_{2}, a_{3}\right]=$ [.02,.025, .03] and $|M|=2$, then (14) is not satisfied; hence, no such asymmetric Nash equilibrium exists.

\section{Competing interests}

The authors declare that they have no competing interests.

\section{Authors' contributions}

YS and GK established the problem formulation, performed false attestation analysis and carried out numerical experiment and analysis. The other authors critiqued the paper; and YS and GK modified the paper accordingly. All authors read and approved the final manuscript.

\section{Acknowledgements}

This research is supported by grant NSF CCF-1228717

\section{Author details}

${ }^{1}$ Department of Computer Science and Engineering, The Pennsylvania State University, University Park, State College, PA 16802, USA. ${ }^{2}$ Department of Electrical Engineering, The Pennsylvania State University, University Park, State College, PA 16802, USA. ${ }^{3}$ Electrical Engineering, Arizona State University, Tempe, AZ 85287, USA. ${ }^{4}$ Department of Computer Science, University of California, Davis, CA 95616, USA.

Received: 15 December 2014 Accepted: 25 February 2015 Published online: 24 March 2015

\section{References}

1. AK David, F Wen, Strategic bidding in competitive electricity markets: a literature survey. Power Engineering Society Summer Meeting. 4, 2168-2173 (2000)

2. N Fabra, N-H von der Fehr, D Harbord, Designing electricity auctions, RAND Jounral of Economics. 31, 23-46 (Spring 2014)

3. EJ Anderson, $\mathrm{HXu}$, Supply function equilibrium in electricity spot markets with contracts and price caps. Journal of Optimization Theory and Applications. 124(2), 257-283 (Dec. 2005)

4. AJ Wood, BF Wollenberg, Power generation operation and control, 2nd edn. (John Wiley \& Sons, Inc., 605 Third Avenue, New York, NY, 1996), pp. 108-109

5. G Wang, SV Shanbhag, S Meyn, in Proc. IEEE CDC. On Nash equilibria of duopolistic power markets subject to make whole uplift (IEEE, Maui, Dec. 2012). doi:10.1109/CDC.2012.6425983

6. M Roozbehani, M Dahleh, S Mitter, in Proc. IEEE SmartGridComm. Dynamic pricing and stabilization of supply and demand in modern electric power grids (IEEE, Gaithersburg, MD, 4-6 Oct. 2010)

7. AH Mohsenian-Rad, A Leon-Garcia, Distributed internet-based load altering attacks against smart power grids. IEEE Trans. on Smart Grid. IEEE. 2(4), 667-674 (2011)

8. M Alizadeh, A Scaglione, A Goldsmith, G Kesidis, in Proc. IEEE CDC. Scalable management of large populations of flexible appliances (IEEE, Los Angeles, 2014). Longer version to appear in IEEE Trans. Power Systems (TPWRS)

9. H Lu, G Pang, G Kesidis, in Proc. IEEE SmardGrid Comm. Automated scheduling of deferrable PEV/PHEV load by power-profile unevenness (IEEE, Vancouver, Oct. 2013)

10. C Chen, S Kishore, Z Wang, M Alizadeh, A Scaglione, in Proc. IEEE Power and Energy Society (PES) General Meeting. A Cournot game analysis on market effects of queuing energy request as demand response (IEEE, San Diego, CA, July 2012) 
11. MG Vaya, G Anderson, in Proc. Int'l Conf. on European Energy Market (EEM). Optimal bidding strategy of a plug-in electric vehicle aggregator in day-ahead electricity markets (IEEE, Stockholm, May 2013)

12. Duke utility bill tariff (2012). http://www.considerthecarolinas.com/pdfs/ scscheduleopt.pdf

13. Fort Collins Coincident Peak (2013). http://www.fcgov.com/utilities/ business/rates/electric/coincident-peak

14. D Bienstock, M Chertkov, S Harnett, Chance-constrained optimal power flow: risk-aware network control under uncertainty. SIAM Review. 56(3), 461-495 (2014)

15. B Bobbs, Linear complementarity models of Nash-Cournot Competition in bilateral and POOLCO Power Market. Utilities Policy. 5(3/4), 194-202 (1996)

16. SD Ledgerwood, PR Pfeifenberger, Using virtual bids to manipulate the value of financial transmission rights. The Electricity Journal. 26(9), 9-25 (2013)

17. C LoPrete, WW Hogan, Manipulation of day-ahead electricity prices through virtual bidding. IAEE Energy Forum. (IAEE, Cleveland, OH, 2014), pp. 31-32

18. G Kesidis, C Griffin, DJ Miller, A marketplace game with neither distribution costs nor distribution-capacity constraints. PSU CSE Dept. Technical Report. CSE-13-013 (Nov. 2013)

19. Y Jin, G Kesidis, Dynamics of usage-priced communication networks: the case of a single bottleneck resource. IEEE/ACM Trans. Networking. IEEE Press, Piscataway, NJ, USA. 13(5), 1041-1053 (2005)

20. JS Shamma, G Arslan, Dynamic fictitious play, dynamic gradient play, and distributed convergence to Nash equilibria. IEEE Trans. Auto. Contr. 50(3), 312-327 (2005)

21. RD Zimmerman, CE Murillo-Sánchez, RJ Thomas, MATPOWER: steady-state operations, planning and analysis tools for power systems research and education. IEEE Trans. Power Syst. 26(1), 12-19 (2011). See also http://www.pserc.cornell.edu/matpower

22. Y Shan, J Raghuram, G Kesidis, C Griffin, K Levitt, DJ Miller, J Rowe, A Scaglione, Generation bidding game with flexible demand. Arxiv.org Technical Report. 1408.6689 (Aug. 2014). Available at http://arxiv.org/ abs/1408.6689

23. IEEE 14-Bus Power-Flow Test Case - System Data. http://www.ee. washington.edu/research/pstca/pf14/pg_tca14bus.htm

24. Y Sasaki, K Kijima, Hypergames and Bayesian games: a theoretical comparison of the models of games with incomplete information. J Syst Sci Complex. 25, 720-735 (2012)

25. S Bhattacharya, K Kar, JH Chow, A Gupta, in Proc. Int'l Conf. on Network Games, Control and Optimization (NeTGCoop). Progressive second price auctions with elastic supply for pev charging in the smart grid (Trento, Italy, Oct. 2014)

26. $X$ Zhou, $\mathrm{H}$ Zheng, in Proc. IEEE INFOCOM. Trust: a general framework for truthful double spectrum auctions, (2009), pp. 999-1007

\section{Submit your manuscript to a SpringerOpen ${ }^{\circ}$ journal and benefit from:}

- Convenient online submission

- Rigorous peer review

- Immediate publication on acceptance

- Open access: articles freely available online

- High visibility within the field

- Retaining the copyright to your article

Submit your next manuscript at $\boldsymbol{\nabla}$ springeropen.com 